\title{
AUGMENTED REALITY AND ARTIFICIAL INTELLIGENCE TO CREATE INNOVATIVE SOLUTION SISOM
}

\author{
LUCIANO DI DONATO \\ Inail, Italy
}

\begin{abstract}
Italian national plan "Industry 4.0" fosters technological innovation in terms of investments and productivity by means of fiscal incentives. Industry 4.0 is an opportunity to develop technologies and assets aimed at achieving advantages such as greater production flexibility, reduced manufacturing lifecycle times and increased competitiveness. In this context, Inail, and in particular Lab 2 of Department of Technological Innovation (DIT), executed research activities on machines and work equipment to analyze new technologies, such as Augmented Reality (AR) and Artificial Intelligence (AI), as well as additive technologies and collaborative robots, from a safety perspective. This paper presents the results of one research project called "Intelligent System for the Safety of Machine Operator" (SISOM), promoted by Inail through a call for bids in collaboration - BRIC 2015 ID 16. This research can be regarded as a first step of analysis of an innovative solution, specifically applied to a machine in the food industry, for the safety of operators.
\end{abstract}

Keywords: Industry 4.0, Augmented Reality, Artificial Intelligence, safety, machine.

\section{INTRODUCTION}

In the framework of Industry 4.0, Inail, and in particular DIT Laboratory 2 Machine and Work Equipment, plans and executes research activities focused on the safety of machines and work equipment. These activities analyze the safety issues related to the use of new enabling technologies to test their value and effectiveness.

Technology innovation is an economic opportunity, nevertheless it is important to perform experimental activities to anticipate and solve emerging issues that can originate from the integration between production technologies and new enabling technologies.

All these activities are required to provide appropriate solutions to the issues that threaten the health and safety of worker.

In this paper the results of a project entitled "Intelligent System for the Safety of Machine Operator" (SISOM) will be described. DIT Laboratory 2 Machine and Work Equipment took over the scientific responsibility of this project in collaboration with the Universities of Parma, Pisa, Genoa and Reggio Calabria. SISOM resulted in a new prototype solution for improving operator's safety, using AR and AI, applied to a machine in the food industry.

The solution delivered will be presented together with its test on field, as a new training model, to decrease the possibility of human-error related accidents.

\section{REFERENCE FRAMEWORK}

The solution realized with SISOM project was developed after a feasibility study, detailed description in [1], for a fruit extractor machine. This machine, in fact, is often used by seasonal operators to improve the information and training program for operators in the food industry. Moreover, the tool realized could be directly adopted to support operator's activities on field.

During the research, contents from manufacturer instructions about the execution of an ordinary maintenance task, characterized by significant risks for operator, were implemented 


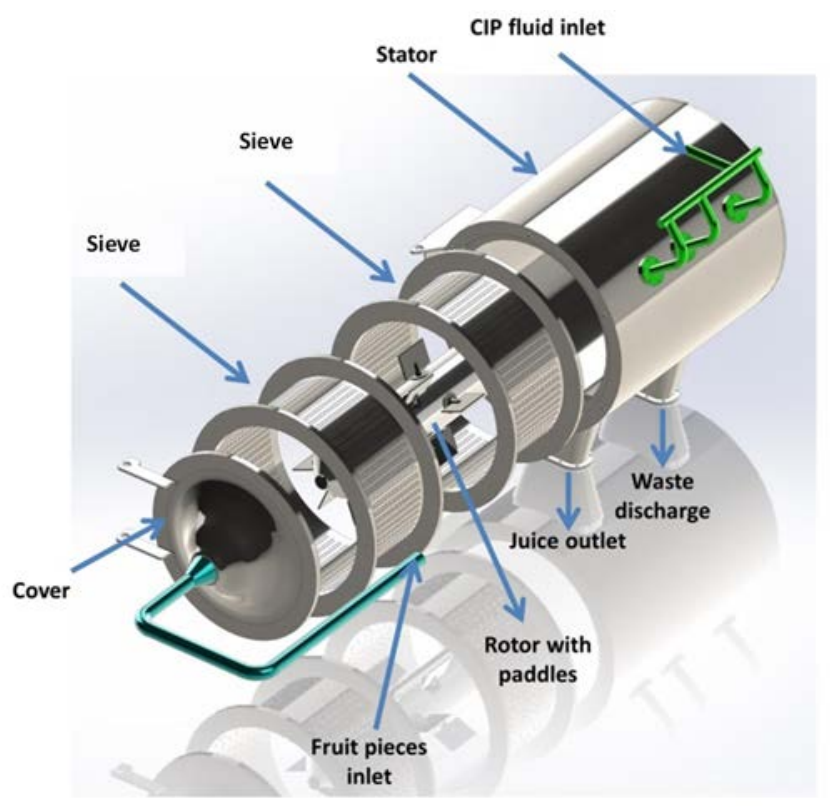

Figure 1: Main components of the fruit extractor.

in AR. More in detail, the task was the cleaning and/or substitution of the porous sieves of the machine, called baskets (see Fig. 1).

This task of cleaning and/or substitution of the porous sieves mainly consists in:

1. stopping the machine,

2. removing two sieves,

3. cleaning them with a water jet,

4. placing the sieves inside the rotor correctly,

5. starting again the machine.

Moreover, the operator has to change the sieves if they are damaged or if the product changes and he has to choose the right sieves for the product treated.

Changing the sieves ensures the production line efficiency is kept constant and should avoid, at the same time, an excessive wear of the paddles necessary to move the product (see Fig. 1), and of the sieves themselves, due to an accumulation of fruit residues in the stator chamber.

This specific task was chosen, among the whole set of maintenance operations that can be carried out on this machine, because it is relatively frequent. If the production line is working at full capacity, this task has to be performed at least every 12 hours, according to the instructions of the machine manufacturer.

Before the execution of the operations described above, the machine is normally working reaching very high temperatures, so that it has to be cooled. Consequently, the operator can come into contact with very hot product (up to $85-95^{\circ} \mathrm{C}$ ) as well as with moving parts (the paddles). The machine manufacturer evaluated and managed these risks selecting technical 


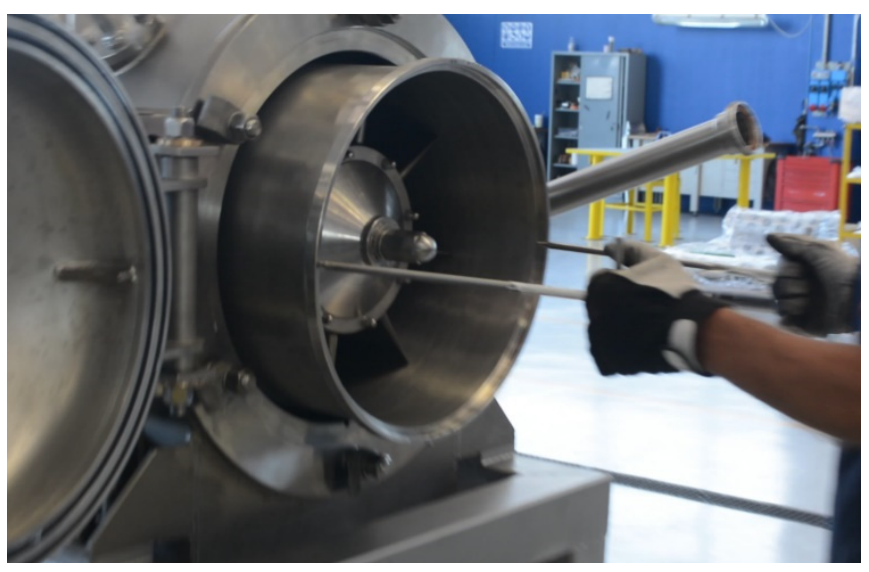

Figure 2: Extraction of the paddle.

and procedural measures that the operator should follow. Instructions for use contain also information and recommendations about these risks.

For instance, the machine manufacturer managed the risk of getting in touch with hot parts of the machine providing two threaded rods with handles (see Fig. 2) that are screwed in special points created on the sieves to keep the operator away from the hot elements and requiring personal protective equipment (PPE), such as gloves, for the execution of this task. Gloves are necessary also for the residual risk to touch the hot external pipes of the machine.

AR technology and vocal assistant can make information available during the execution of dangerous tasks like this - for instance about the use of rods and dressing of PPE - helping the operator lowering the likelihood of accidents in the man-machine interface.

\section{SISOM PROTOTYPE}

The solution realized with SISOM project was designed to be finally installed on a mobile system, such as a smartphone or a tablet. The camera of the mobile device, through an app called exactly SISOM, recognizes a marker installed on the machine next to the control panel. It was not possible to place markers directly on the machine or on any of its elements/parts which could compromise its functioning. For example, it was not possible to install a marker on the sieve, as the sieve is in direct contact with the food product. The fruit is at hot temperature, meaning that the marker would be subject to relevant thermal excursions.

After recognizing the tag, a voice manager subsystem starts and processes the user request converting both the vocal request in text and the text answer in voice form. The user request, after being processed, is sent to a search manager. This search manager starts the functions necessary to process feedback contents.

The AR contents were developed using the 3D rendering of the machine (see Fig. 3 ) that was created from some videos and pictures from manufacturer instructions. 


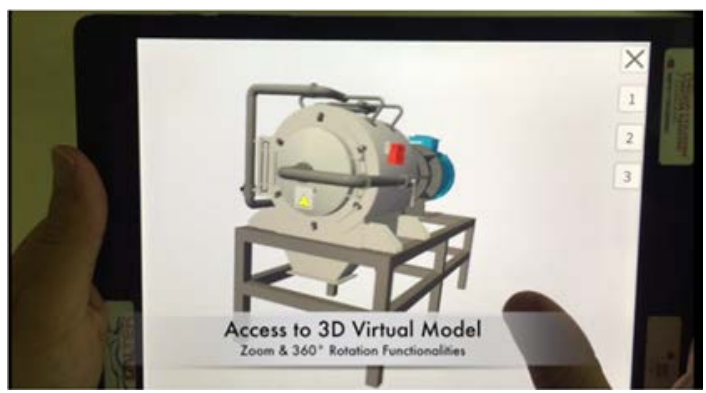

Figure 3: 3D virtual model of the fruit extractor.

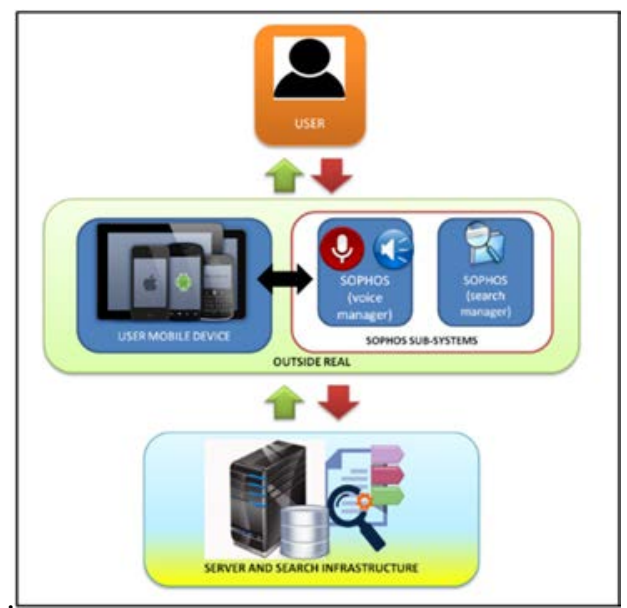

Figure 4: SISOM technical architecture.

\subsection{The AR solution and the AI driven personal assistant}

The AR solution guides the operator, during the maintenance task selected, using an interactive personal assistant that allows to enter and search for AR contents. This AI driven personal assistant was developed with a software named Sophos-MS.

This Sophos-MS software consists in a navigator of knowledge and is an artificial intelligence system as it is able to answer to user queries. In particular, Sophos-MS consists in software components and related infrastructure realized with a technical architecture allowing the access to information from and to the mobile device (see Fig. 4).

Sophos-MS is inclusive of:

- recognition algorithms,

- voice-text conversion systems,

- search functions activated by user requests,

- content indexing,

- matching algorithms and correlation to provide user suggestions. 
Research features have been implemented through a search engine called Lucene, an open-source solution. This solution supports the operator in requesting and collecting information about the task he has to carry out, thus coaching him in performing the required activities and reducing the occurrence of accidents related to the man-machine interface.

\subsection{Information classification}

The design of such an interactive personal assistant requires a further preliminary step. This step mainly consists in the selection and classification of the information that can be acquired by means of keywords or questions that the employee can ask when carrying out the task supported by the AR system. Indeed, the AI driven personal assistant works using a natural language user interface to answer questions and make recommendations, while retrieving the relevant information from the set of keywords.

Some keywords were easily identified from a set of sample questions, for instance "Where is button 13? What is button 13 for?", "Where is the electric panel?", or "What is the correct procedure for turning off the product flow". Categorizing the data collected means to give them a sort of tag ("button 13", "electric panel", "product flow"), so that the personal assistant can search the answer to the employee questions by screening these tags.

\section{TEST}

The first prototype of the solution realized with SISOM project was tested at the manufacturer plant. During the tests, the same maintenance task was carried out by 24 students without a previous knowledge of this fruit extractor machine. The students were divided into two groups: one group, only traditionally trained, directly using the SISOM mobile device during operations, and the other group with an additional training on the SISOM solution.

As output, it was measured:

- the execution time of the maintenance task,

- the number of errors made during the execution of the task.

The outcomes of these tests highlighted that the use of the SISOM solution has the potential to decrease the number of errors made by an employee. Videos turned out to be the feature of the AR solution that was most effective in avoiding errors. Moreover, the test results showed that the execution time of the maintenance task does not necessarily decrease when using the SISOM solution. In some occasions, it was not immediate for the student to interact with some features of the solution (e.g. the AI driven voice assistant).

Indeed, although the tests carried out provided some preliminary findings about the usefulness of this new enabling technology, the limited number of people involved prevented the possibility of providing statistical evidence of the results obtained. Moreover, a natural future step of this research is to quantify the benefits resulting from the use of this solution.

\section{CONCLUSION}

The solution realized with SISOM project has highlighted some aspects that need to be properly considered to obtain benefits in operator safety through the adoption of new enabling technologies of the so-called Industry 4.0, also defining a new effective training model for the specific context under consideration.

Surely to establish the functional requirements of the AR solution component, it is necessary to start with a deep analysis of the information that the employee may need during the execution of his task. 
This research project has also studied a reference methodology to analyze the risks related to human behavior. This methodology could be used as groundwork for an effective application of AR and AI technologies on hazardous activities. Next research activities will study in deep, the potentiality and critical issues of implementing an AI system integrated with the control system of the machine and process data, so that the employee could retrieve, for instance, information about the next production lot and therefore on the kind of sieve to place on the fruit extractor.

In designing and building a machine and writing its operating instructions, the manufacturer must consider, not only the intended use of the machine, but also the reasonably foreseeable misuse. Developing additional systems that can guide human behavior by performing less mistakes is a very interesting opportunity, but it must be well-managed to ensure safer operation.

\section{REFERENCES}

[1] Spanu, S., Bertolini, M., Bottani, E., Vignali, G., Di Donato, L., Ferraro, A. \& Longo, F., Feasibility study of an Augmented Reality application to enhance the operators' safety in the usage of a fruit extractor, I3M - Cipro, Sep. pp. 26-28, 2016.

[2] Besbes, B., Collette, S. N., Tamaazousti, M., Bourgeois, S. \& Gay-Bellile, V., An interactive augmented reality system: a prototype for industrial maintenance training applications. In Mixed and Augmented Reality (ISMAR), IEEE International Symposium, pp. 269-270, 2012.

[3] Carmigniani, J., Furht, B., Anisetti, M., Ceravolo, P., Damiani, E. \& Ivkovic, M., Augmented reality technologies, systems and applications. Multimedia Tools and Applications, 51(1), 341-377, 2011.

[4] Geller, E. S., Behavior-based safety in industry: realizing the large-scale potential of psychology to promote human welfare. Applied \& Preventive Psychology, 10, pp. 87105, 2001.

[5] Servàn, J., Mas, F., Menéndez, J. L. \& Ríos, J., Assembly work instruction deployment using augmented reality. In Key Engineering Materials, 502, pp. 25-30, 2012.

[6] Van Krevelen, D.W.F. \& Poelman, R., A survey of augmented reality technologies, applications and limitations. The International Journal of Virtual Reality, 9(2), 1-20, 2010.

[7] Vlahakis, V., Ioannidis, N., Karigiannis, J., Tsotros, M., Gounaris, M., Almeida, L., Stricker, D., Gleu, T., Christou, I. T. \& Carlucci, R., ARCHEOGUIDE: First results of an augmented reality, mobile computing system in cultural heritage sites. Proceedings of the 2001 Conference on Virtual Reality, Archeology, and Cultural Heritage (VAST 2001), 131. DOI:10.1145/584993.585015.

[8] Rios, H., González, E., Rodriguez, C., Siller, H. R. \& Contero, M., A Mobile Solution to Enhance Training and Execution of Troubleshooting Techniques of the Engine Air Bleed System on Boeing 737. Procedia Computer Science, 25, 161-170, 2013.

[9] Braglia, M., Di Donato, L., Gabbrielli, R. \& Marazzini, L., The house of safety: a novel method for risk assessment including human misbehaviour. Submitted for publication Reliability Engineering and System Safety. 